\title{
Ein seltenes Osteom der Stirnhöhle und Siebbeinzellen.
}

\author{
Von Dr. Stolpe, Quedlinburg a. H.
}

Mit 7 Abbildungen.

Osteome der Nebenhöhlen sind seltene Tumoren. In der Literatur bis rgIo hat Boenninghaus nur I98 Fälle gefunden.

Es lohnt sich deshalb, den von mir im Jahre IgI 8 im Felde behandelten und operierten Fall näher zu betrachten.

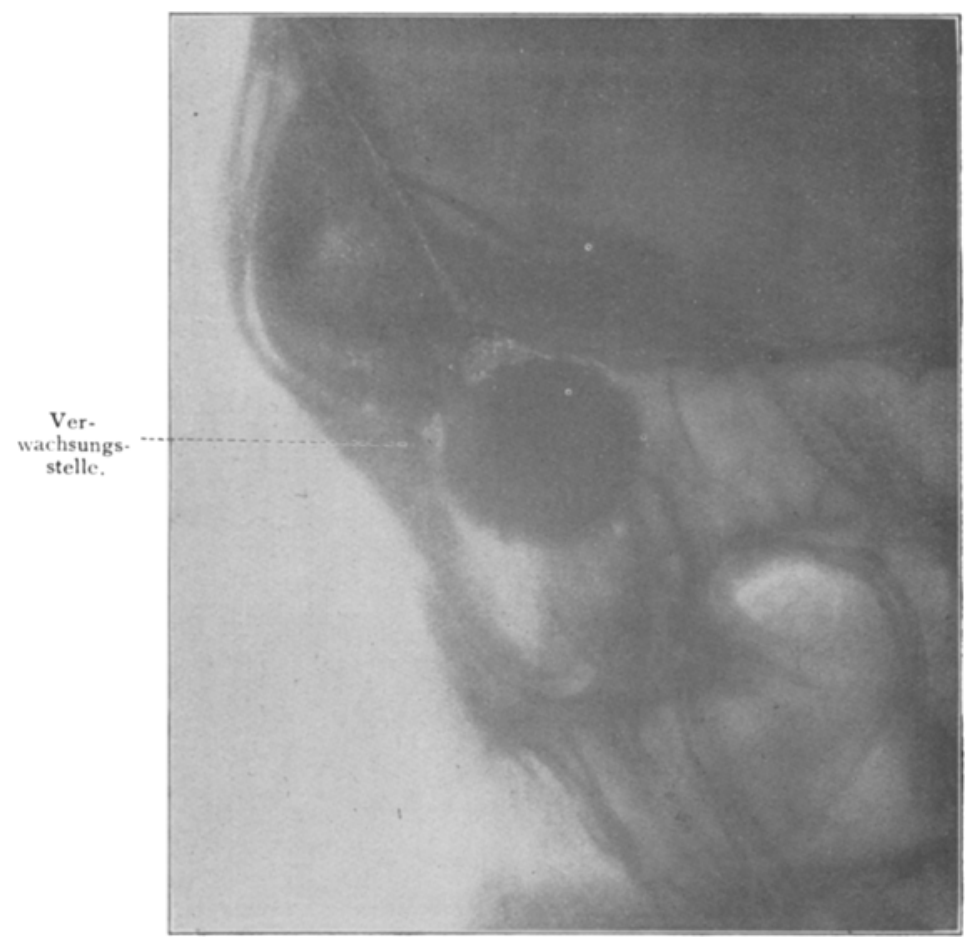

Abb, I. Seitliche Röntgenaufnahme (Osteom).

Anamnese. Franz K., Infanterist, Feldrekrutendepot 4, geb. I4. VI. 97. Familie o. B. Mit I4 Jahren Blinddarmentzündung. Mit is Jahren Sturz auf den Kopf mit leichter Verwundung oberhalb des linken Auges. Sonst bisher gesund. Nikotin- und Alkoholgenuß immer sehr mäßig. Geschlechtliche Ansteckung verneint.

Am 22. I. I8 plötzlich Unwohlsein. Kopfschmerzen in der Stirngegend. 
Am 27. I. Schüttelfrost, Fieber 39, I. Erbrechen. Schwellung der linken Gesichtshälfte, besonders des linken Oberlides. Das linke Auge konnte nur wenig geöffnet werden. Leichtes Flimmern links. Deshalb Aufnahme im bayr. Feldlazarett 12 am 28. I. I8.

Hierselbst Befund: Linke Gesichtshälfte wenig geschwollen, leicht gerötet; linkes

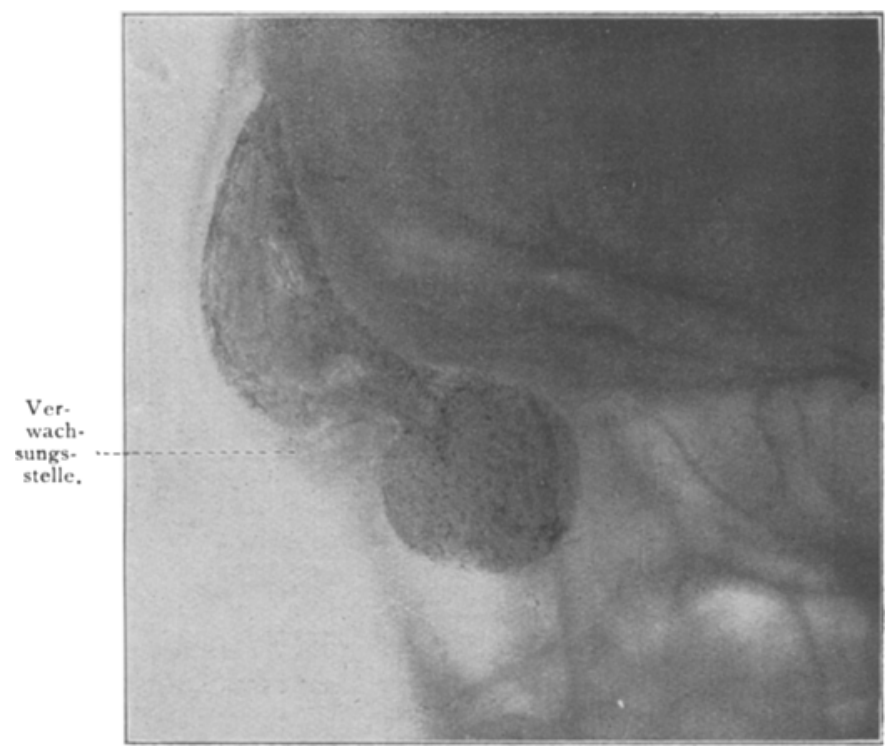

Abb. 2. Seitliche Röntgenaufnahme (Osteom).
Oberlid deutliche Schwelluingo und Ptosis. Bindehaut des linken Auges leicht gerötet. Pupillen trotz Lichteinfall erweitert. Supraorbitalgegend, noch erhalten. besonders links druckschmerzhaft. Keine Drüsenschwellung. Nackengegend frei.

Puls, Herz, Lunge, Leib, Reflexe, Urin o. B. 30. I. Schwellung hat abgenommen, Druckempfindlichkeit Nase o. B., etwas schleimiges Sekret. Inneres Auge und Hintergrund o. B. Sehvermögen des linken Auges nicht gestört. Krankheits: bezeichnung: Verdacht auf Stirnhöhlenerkrankung unter Beteiligung des Periosts.

I. II. I8 dem bayr. Feldlazarett i I überwiesen. Befund unverändert. Keine Temperatursteigerung.

Abb. 3. Fronto-okzipitale Röntgenaufnahme. (Osteom). 
3. II. I8. Starke Kopfschmerzen über beiden Stirnhöhlen und über der linken Schläfe, sonst Befund unverändert.

Röntgenaufnahme:

I. frontal: scharf begrenzter diffuser Schatten in der Gegend der linken Siebbeinzellen, der linken Stirnhöhle und des medialen Teiles der rechten Stirnhöhle.

2. seitlich: diffuser Schatten in der Gegend der linken Siebbeinzellen. und Stirnhöhle.

Deshalb von Prof. Pagenstecher. Überweisung am 5. II. I8 an die Ohren-, Nasen-, Hals-Armeestation (des bayr. Kriegslazarett 26B), deren ordinierender Spezialarzt ich damals war.

Befund. Mittelgroßer Mann, mittelstarker Knochenbau, leidlich gut entwickelte Muskulatur. Mittelguter Frnährungszustand.

Das Auge links steht in der Horizontalebene etwas tiefer als rechts. Geringe Schwellung unterhalb der linken Augenbraue; hier läßt sich der Finger nicht so tief eindrücken, wie rechts; ebenso am Übergange des Bodens der Stirnhöhle zum vorderen Teile der medialen Augenhöhlenwand, wobei man allenthalben das Gefühl einer festen diffusen knochenartig resistenten Hervorwölbung hat. Keine entzündlichen Erscheinungen, keine Drüsenschwellungen. Nase, außer etwas schleimigen Sekret links, o. B. Keine Temperatursteigerung, Allgemeinbefinden gut. Doppelsehen. Augenbefund (Prof. Crusius, Augen. station) 5. II. I8. Linke Lidspalte Spur enger wie rechts. Pupillen R. Spur $>$ L. Reaktion + . Ophth. Befund o. B.
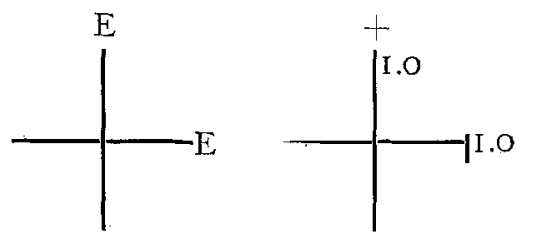

$$
\begin{aligned}
& \text { R. }{ }^{6 / 6} \\
& \text { L. } 1 / 10
\end{aligned}
$$

Gesichtsfeld peripher völlig frei, für Weiß und Farben jederseits. Dagegen ist links ein relatives zentrales Skotom für Farben nachweisbar und daraus erklärt sich auch die Herabsetzung der zentralen Sehschärfe links.

Diagnose: Links Retrobulbäre Sehnervenschädigung. Konvergenzinsuffizienz links.

Röntgenaufnahme (Privatdozent Dr. Alwens, Röntgenstation) Io: II. I8.

Die trarsversale (seitliche) Aufnahme war für mich resp. für die auszuführende Operation insofern von großer Wichtigkeit, als ich aus ihr deutlich ersehen konnte, da $B$ es sich hier um einen Nebenhöhlentumor handelte, der sicherlich nicht von der AuBenwand der Stirnhöhle exostosenartig, sondern von der Innenwand derselben sich entwickelt hatte. Ferner konnte aus der seitlichen Aufnahme mit Sicherheit gesagt werden, daß kein bösartiger Tumor vorlag. Dagegen sprach, daB er ausgußartig die Stirnhöhle ausfüllte, seine ebenmäßige glatte Oberfläche und der negative Befund von Drüsenschwellungen; die Oberfläche läuft allenthalben der Innenwand der Stirnhöhle, getrennt durch einen etwa $1 / 2 \mathrm{~mm}$ breiten Zwischenraum parallel; die Benignität gilt auch für den durch den Ductus naso-frontalis, resp. die Infundibularzellen sich bis tief in die vorderen Siebbeinzellen fort- 
setzenden mit einem anscheinend breiten Stiel verbundenen kugeligen Fortsatz des Stirnhöhlentumors. Man sieht, wie er hier die dünnen leichter nachgiebigen maschenartigen Wände der Siebbeinzellen verdrängt hat und daß hier nicht mehr eine die Geschwulst umgebende Wand, wie eine solche dickwandige widerstandsfähigere der Stirnhöhle besteht, sondern daß sie, wenn überhaupt eine besteht, mindestens von einer derartig, vielleicht seidenpapierdünnen Beschaffenheit ist, daß sie sich im Bild nicht mehr dokumentieren konnte; auch ist es möglich, daß nur noch als äußere Wand, eine ganz dünne Schicht der Iamina papyracea bestand, die sich aber wiederum im frontalen Bilde besser präsentiert haben würde.

An dem medialen Teile des Bodens der linken Stirnhöhle, etwa

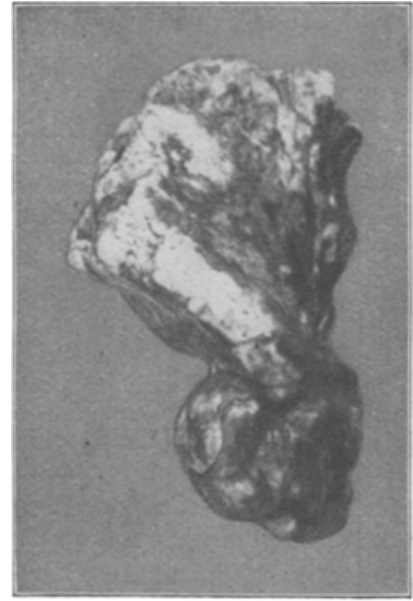

Abb. 4 (natürl. Größe). Photographische Aufnahme. Tumor nach Entfernung in derselben Lage wie Abb. I und 2 zeigt.

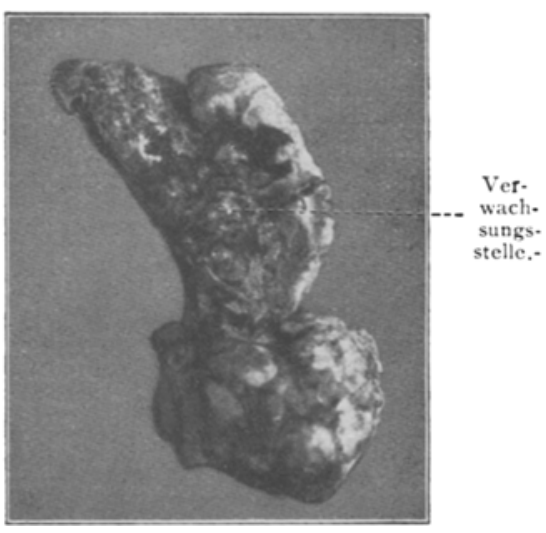

Abb. 5 (natürl. Größe). Photographische Aufnahme. Tu-. mor nach Entfernuing in derselben Lage (von vorn) wie Abb. 3 (kariöse Stelle sichtbar).

zwischen Septum und diesem, scheint der Tumor diffus in die Knochenwand überzugehen entsprechend dem an dieser Stelle sich befindlichen, nicht mehr scharf begrenzten, nicht mehr von der Außenwand durch einen Hohlraum getrennten Schatten. Hier ist etwa $4 \mathrm{~mm}$ breit ein Zwischenraum zwischen Wand und Tumor nicht nachweisbar und deshalb vermutete ich, daß der Tumor von dieser Stelle aus seinen Ursprung genommen habe.

Keilbeinhöhle und Kieferhöhle sind frei. In dem stereoskopischen seitlichen Bilde konnte man all die erwähnten Beschaffenheiten mit noch größerer Präzision deutlich erkennen.

Die frontale Aufnahme läßt durch die überlagernden Hinter- 
hauptsknochen einen weniger deutlichen Schatten erkennen, der bis in die rechte Stirnhöhle zum Teil reicht, ferner in die linken Siebbeinzellen und dort die Lamina papyracea ein wenig lateral hervorwölbt.

Diagnose: Differentialdiagnose zwischen Osteome und zystöser Xebenhöhlenpolypenbildung.

Die umgrenzte Schattenbildung des Röntgenbildes und die Erscheinungen vom Auge aus berechtigten mich nicht zur alleinigen bestimmiten Diagnose eines Osteoms, da ich zur gleichen Zeit bei einem anderen Patienten Stirnhöhlen- und Nasenpulypen nasal entfernt hatte, die im Röntgenbilde ebenfalls einen umgrenzten Schatten zeigten und die eine bedeutende Herabsetzung der Sehschärfe durch zentrales Skotom hervorgerufen hatten.

Nach Entfernung zahleicher Polypen war die Stirnhöhle im Röntgen-

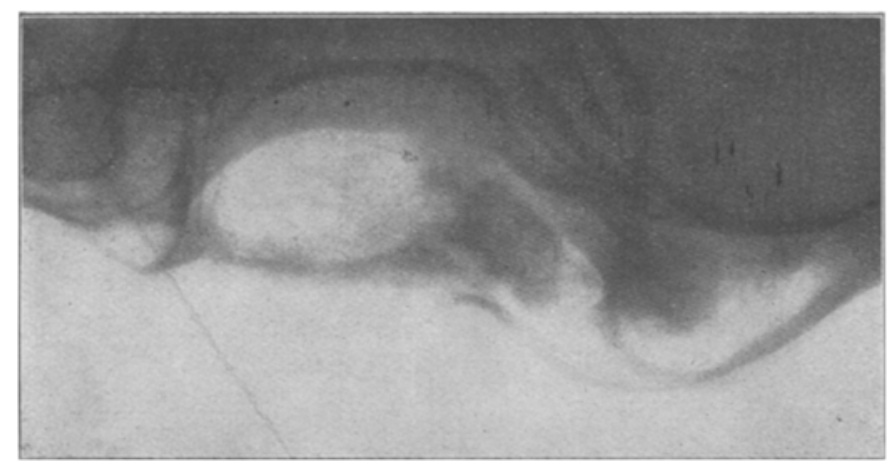

Abb. 6.

Seitliche Röntgenaufnahme. Nasen- und Stirnhöhlenpolypen.

bilde frei und die Sehschärfe fast normal wieder hergestellt (siehe Abbildung 6 u. 7).

Nach Einwilligung des Patienten zur Operation wird sie am I5. II. I8 ausgeführt.

Operation: Vorher Morphiuminjektion. Tampon in linke Nasenhölile und Nasenrachenraum. Chloroformäthernarkose. Killianscher Haut- und Periostschnitt. Zurückschaben des Periostes. Schon nach dem ersten Hammerschlag auf den Meißel über dem medialen Teil der frontalen Wand liegt ein Knochentumor von elfenbeinartiger Beschaffenheit und etwas knolliger Oberfläche vor. Nach weiterem Abtragen der vorderen Stirnhöhlenwand erweist es sich, daß der Tumor fast die ganze Stirnhöhle ausgußartig ausfüllt, aber am medialen Teile des Margo supraorbitalis fast zwei Drittel des Margo und den vorderen medialen Teil des Stirnbodens am Übergange zu den Siebbeinzellen völlig ersetzt.

Etwas außerhalb der Mitte des Margo supraorbitalis sieht man eine 
I $\mathrm{cm}$ lange vertikal verlaufende, I $\mathrm{mm}$ breite kallusartige Leiste. Der Teil des Tumors, der in der Gegend des Septum intrafrontale liegt, ist usuriert mit polypenartigen, ödematösen und zystenartigen kleinen Schleimhautwucherungen und Granulationen besetzt. Vom Septum ist nur noch eine kleine, etwa I mm dicke Crista an der fazialen Wand vorhanden, wo der Knochen ebenfalls usuriert eitrig infiltriert und mit dem Tumor verwachsen ist. Von hier aus sieht man auch mehrere Polypen in die rechte Stirnhöhle hineinragen. Etwa $1 / 2 \mathrm{ccm}$ freien Eiters. Der Tumor wird durch Meißeln von der vorderen Innenwand losgelöst.

Das äußere Drittel der linken Stirnhöhle ist frei. Nun wird das orbitale Periost zurückgeschabt, die Periorbita losgelöst und samt Augapfel

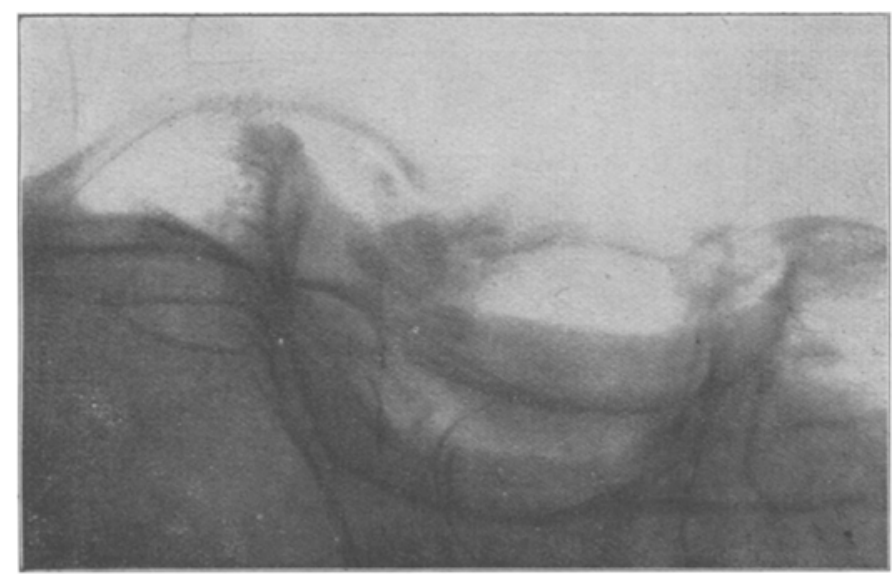

Abb. 7. Seitliche Röntgenaufnahme nach Entfernunz, der Nasenund Stirnhöhlenpolypen.

nach auswärts gezogen, so daß man jetzt eine gute Utbersicht über das Dach und die mediale Wand der Orbita gewonnen hat. Die Lamina papyracea ist kugelig hervorgewölbt und seidenpapierdünn; nảch $\mathrm{Ab}$ tragung derselben liegt ein kirschgroßer, ebenfalls elfenbeinartiger Tumor von knolliger, im allgemeinen glatter Oberfläche vor und ist mit dem lateralen unteren Teile des Tumors der Stirnhöhle verbunden. Nach Entfernung der ganzen Knochengeschwulst mit Hebel und Zange sieht man, daß der kugelige Teil bis zur vorderen Wand der Keilbeinhöhle reichte, das Dach der Kieferhöhle medial durch Druck des kugeligen Teiles z. T. atrophisch usuriert resp. geschwunden und hierdurch eine Inspektion der Kieferhöhle ermöglicht ist. Vor der Lamina cribrosa links ist eine etwa linsengroße Stelle der Basis cranii druckatrophisch defekt. Die Dura liegt hier leicht gerötet, glatt vor. Die kariösen entzündlichen exostosenartigen Reste an der Verwachsungsstelle des 
Osteoms mit der Diploe liegen jetzt frei und übersichtlich vor, werden mit Meißel bis zur Glättung entfernt.

Zum SchluB Entfernung einiger granulöser Siebbeinzellen mit dem scharfen Löffel. Herstellung einer breiten Kommunikation mit der linken Nasenhöhle.

Einführung eines Tampons in die rechte und linke Stirnhöhle, in die Tiefe der Siebbeinzellen und von hier aus in die Nasenhöhle. Naht des äußeren Drittels der Schnittwunde. Verband. Entfeinung des Tampons aus dem Nasenrachenraum.

Verlauf. Die Heilung wurde leider durch ein Erysipel des Kopfes und Rumpfes und durch einen linksseitigen Kniegelenkserguß etwas verzögert.

Heilung des Erysipels am I. IV. I8, des Ergusses am 25. IV. I8.

Am I. V. 18 hatte sich die Operationshöhle durch gute Granulationen bedeutend verkleinert. Von der linken Nasenhöhle aus gelangt man noch mit dicker Sonde in der Höhe des mittleren Nasenganges in die Operationshöhle. Äußeres Auge o. B. Kein Doppelsehen. Besserung der zentralen Sehschärfe. Gutes Allgemeinbefinden. Gewichtszunahme.

Patient wird auf Wunsch einem Heimatslazarett überwiesen.

Betrachtung. Aus den in der Literatur zusammengestellten Fällen von Nebenhöhlentumoren ist $z u$ ersehen, da $\beta$-dieselben meist von der Innenwand entstehen und vorwiegend die Stirnhöhlen befallen. Aber für Stirnhöhlenosteome ist die Ausgangsstelle meist nicht die Stirnhöhle selbst, sondern die Infundibularzellen resp. der vordere Teil der Siebbeinzellen.

Bevor ich mich für den Ursprungsort des Osteoms in dem vorliegenden Falle entscheide, möchte ich ein paar Worte über die Entwickelungsgeschichte der Osteome sagen. In der Entwickelungszeit entstehen durch Resorptions- und. Wachstumsvorgänge unter der Schleimhautauskleidung der Nasenhöhle an bestimmten Stellen der knorpeligen Seitenwand derselben die Nebenhöhlen der Nase. Die Ossifikation des knorpeligen Siebbeins beginnt an der Lamina papyracea im fünften Monat des Fötallebens. Bisweilen gehen aber die knorpeligen Anlagen nicht zugrunde, sondern proliferieren und bilden mit Vorliebe in der Pubertätszeit Exostosen, die sich auch nach der Stirnhöhle zu ausdehnen und die der Ausgangspunkt für Osteome werden. Stirnhöhlenosteome entstehen aber auch vom Endost; dann entstehen sie wohl meist von der ctrebralen, am seltensten von der vorderen Wand der Stirnhöhle, dicht am Septum.

Von letzterer Stelle hat sich der vorliegende Tumor wohl zweifellos und zwar vom Endost aus entwickelt. Der Fall auf das Stirnbein mit I5 Jahren kann die Gelegenheitsursache zum Wachstum gegeben 
haben; es ist wahrscheinlich, da $B$ damals eine Infraktion oder Fraktur des linken Stirnbeins, als deren Bewies noch die lineare vertikale Callusbildung am Margo supraorbitalis bei der Operation vorlag, bestanden haben mag, die mit e ner Entzündung der Schleimhaut und des Endosts der Stirnhöhle verbunden gewesen ist. Das sich im Anschluß hieran entwickelnde Osteom ist nun während der nächsten Jahre langsam gewachsen, bis es die Stirnhöhle ausgußartig ausgefült hat, ohne sich deutlich manifestierende Symptome zu machen; dann ișt es den Infundibularzellen folgend nach den Siebbeinzellen hin weiter gewachsen und hat dort den kugeligen Tumor gebildet. Als dieser seine jetzige Größe erreicht hatte, traten die ersten Symptome auf, zuerst allgemeiner Art, wie Unwohlsein, Appetitlosigkeit, Erbrechen, Kopfschmerzen, Fieber, Schüttelfrost, dann spezifischer Art, wie Druckempfindlichkeit der Stirnhöhle, Ödem und Ptosis des linken Oberlides. Dazu kamen die Druckerscheinungen innerhalb der Augenhöhle, Gesichtsfeldeinengung durch Druck auf den Nervus opticus, Exophthalmus durch retrobulbären Druck; durch völligen Verschluß des medialen Teiles der Stirnhöhle trat dort ein Stauingskatarrh ein, der einen günstigen Boden für Infektionserreger abgab und zur Ėntzündung resp. Eiterung führte. Intra operationem dokumentierte sich dieselbe an der Verwachsungs- resp. Ursprungsstelle des Osteoms, an der Diploe der frontalen Wand in der Form einer Ostitis. Hier sah man ja bei der Operation den entzündlichen, z. T. in Einschmelzung begriffenen, granulösen, eitrigen Knochen, freien Eiter und Polypen.

Hätte man sich durch die in der letzten Zeit vor der Operation eingetretene Besserung des Allgemeinbefindens und den Stillstand der spezifischen Symptome beeinflussen lassen, mit der Operation noch zu warten, wären sicherlich in kurzer Zeit schwere Komplikationen hinzugekommen. Auf die in die Stirnhöhle hineinragende freiliegende Dura, an der Stelle des Defektes des Bodens der Stirnhöhle, hätte sich der eitrige Prozeß fortsetzen und somit zur Meningitis oder zum Gehirnabszeß führen können oder durch Übergreifen auf den Orbitalinhalt zur Orbitalphlegmone. Durch weiteres Wachstum des kugeligen Tumors wäre eine Atrophie des Nervus opticus mit Erblindung eingetreten und eine Stauung der Orbitalvenen, die wiederum einen günstigen Boden für eine Orbitalphlegmone geschaffen hätte.

Mit diesem Falle ist also wieder einmal bewiesen worden, daß Osteome der Nebenhöhlen, sobald sie als solche erkannt sind, sofort zu operieren sind, andernfalls eine Gefahr für das Auge und für das Leben des Patienten eintritt. 